\title{
Médecins pour le Parlement
}

$36 *$ médecins candidats aux élections parlementaires de cet automne, dont 2 femmes, ont répondu à notre appel et nous ont envoyé une brève description de leur personne et de leurs options politiques (voir BMS No 36, pages 183848, et ce numéro, pages 1918-20).

Ces collègues posent leur candidature dans 14 cantons pour 11 partis différents. Avec 11 et 8 candidats respectivement, les cantons de Zurich et de Vaud sont particulièrement bien représentés.

Concernant les partis, le PRD est le plus fortement représenté avec 11 candidats, suivi par le PDC, le PST/POP et les Verts avec 4 candidats chacun. Le PS a trois candidats. Les Libéraux, l'UDC, le PEV et le «Freies Forum» ont chacun 2 candidats. Un médecin se porte candidat pour les Démocrates Suisses, un autre est sans parti.

En tout, se présentent ainsi aux élections 13 praticiens généralistes, 4 chirurgiens, 4 spécialistes de prévention et santé publique, 3 psychiatres, 8 porteurs d'un autre titre de spécialiste et 4 médecins sans titre de spécialiste.

Nos collègues ont une moyenne d'âge de 53 ans (min. 24 ans, max. 73 ans).

Le nombre de médecins briguant un siège au Parlement est élevé et nous en sommes très heureux. L'éventail des partis politiques représentés est aussi très large, ce qui nous réjouit également beaucoup sans nous surprendre pour autant. Il est bien loin le temps où les médecins n'avaient pas besoin de se soucier de politique. Comme est aussi révolue l'époque - si elle a vraiment existé - où le corps médical adhérait à un parti déterminé. A l'instar des articles publiés dans le BMS, les descriptions fournies par les candidats sont très hétérogènes et contiennent, en partie, des opinions contraires sur la politique de la santé et la politique professionnelle. «2 médecins, 3 avis» est un adage qui n'est plus exclusif à la seule pratique de la médecine.

Même si ces candidats ne constituent pas un échantillon représentatif du corps médical, nous en retirons cependant la nette impression que celui-ci s'est éveillé à la politique. En tout cas, les raisons de ne plus vouloir se contenter d'un rôle passif ne lui manquent pas.

Dans ce contexte, on peut se demander comment la FMH doit gérer ces différentes tendances politiques en son sein. Ses structures actuelles conviennent extrêmement bien à une politique classique de maintien des droits acquis. Si elle devait mener un débat d'idées entrant en concurrence, ce qui serait non seulement légitime mais aussi souhaitable vu l'évidente diversité des opinions politiques en présence, les organes concernés de la FMH seraient confrontés à des difficultés énormes, voire insurmontables. Eviter ces difficultés et chercher le consensus par un silence unanime n'est pas une solution satisfaisante à long terme. En ce qui concerne la FMH, la question reste donc ouverte pour le moment.

Mais il me reste au moins la possibilité d'agir en tant que citoyen et d'exercer une influence politique en allant voter!

Markus Trutmann 